\title{
Panniculitis as first manifestation of disseminated cryptococcosis
}

\section{Verónica Rotela, Fátima Agüero, Beatriz Di Martino Ortiz, Mirtha Rodriguez, Oilda Knopfelmacher, Lourdes Bolla}

Department of Dermatology, Clinicas Hospital, Faculty of Medical Sciences, National University of Asuncion, Paraguay

Corresponding author: Prof. Beatriz Di Martino Ortiz, E-mail: beatrizdimartino@gmail.com

\begin{abstract}
Cryptococcosis is an opportunistic fungal infection caused by Cryptococcus neoformans. Encapsulated yeast worldwide distribution, related to pigeon droppings, its main entrance is inhaled. Asymptomatic in immunocompetent, but produces exceptionally pneumonitis and a skin primoinfection. In immunocompromised individuals, it spreads through the blood mainly affecting the central nervous system, skin and bones. We report the case of a 23-year-old woman, veterinary student, chronic insufficient kidney by diffuse glomerulosclerosis of unknown etiology for 2 years, Hypertension and anemia of chronic diseases treated with prednisone, cyclophosphamide boluses monthly, and triweekly hemodialysis; with violet-erythematous nodules on legs and right arm, showing oval birefringent structures, intracytoplasmic PAS positive, yeast, encapsulated, surrounded by a clear halo around a core biopsy of hypodermis, and skin culture positive for Cryptococcus neoformans. Treatment was established with fluconazole $400 \mathrm{mg} / \mathrm{d}$ for two weeks, then $200 \mathrm{mg} / \mathrm{d}$ as crops, with a resolution of skin lesions. Not detected until 12 months of follow-up by Cryptococcus neoformans invasion in other organs.
\end{abstract}

Key words: Paniculitis; Cryptococus neoformans; Cryptococcosis

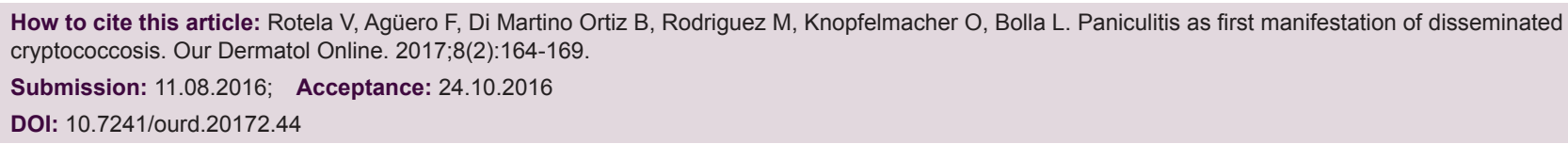




\title{
Paniculitis como primera manifestacion de una criptococosis diseminada
}

\author{
Verónica Rotela, Fátima Agüero, Beatriz Di Martino Ortiz, Mirtha Rodriguez, \\ Oilda Knopfelmacher, Lourdes Bolla
}

Department of Dermatology, Clinicas Hospital, Faculty of Medical Sciences, National University of Asuncion, Paraguay

Corresponding author: Dra. Beatriz Di Martino Ortiz, E-mail: beatrizdimartino@gmail.com

\begin{abstract}
RESUMEN
La criptococosis es una infección micótica oportunista causada por Cryptococcus neoformans, levadura encapsulada de distribución mundial, relacionada con excrementos de palomas. Su principal puerta de entrada es inhalatoria. En inmunocompetentes produce neumonitis asintomática y excepcionalmente una primoinfección cutánea; en individuos inmunodeprimidos, se disemina por vía hematógena afectando principalmente al sistema nervioso central, piel y huesos. Presentamos el caso de una mujer de 23 años, estudiante de veterinaria, insuficiente renal crónica por glomeruloesclerosis difusa de etiología no filiada desde hace 2 años, HTA y anemia de las enfermedades crónicas en tratamiento con prednisona, bolos mensuales de ciclofosfamida y hemodiálisis trisemanal, con nódulos eritematovioláceos en piernas y brazo derecho, observándose estructuras birrefringentes ovaladas, intracitoplasmáticas, PAS positivas, levaduriformes, encapsuladas, rodeadas de un halo claro alrededor de un núcleo redondeado en la biopsia de la hipodermis y con cultivo de piel positivo para Cryptococcus neoformans. Se instauró tratamiento con fluconazol $400 \mathrm{mg} /$ día dos semanas, luego $200 \mathrm{mg} / \mathrm{d}$ según cultivos con resolución de lesiones cutáneas, no detectándose hasta los 12 meses de seguimiento invasión por Cryptococus neoformans de otros órganos.
\end{abstract}

Palabras claves: Panniculitis; Cryptococus neoformans; Criptococosis

\section{INTRODUCCION}

La criptococosis es una nfección micótica oportunista causada por el Cryptococcus neoformans $(\mathrm{CN})$, levadura encapsulada de distribución mundial, relacionada con los excrementos de palomas y murciélagos, frutas, vegetales y tierra.

Su principal puerta de entrada es inhalatoria y en personas inmunocompetentes produce neumonitis asintomática con resolución espontánea y excepcionalmente una primoinfección cutánea.

En individuos inmunodeprimidos, la infección se disemina por vía hematógena afectando a otros órganos, principalmente al sistema nervioso central, la piel y los huesos, sin olvidar que cualquier órgano o tejido es susceptible de dicha invasión.
El sistema nervioso central es, tras los pulmones, el segundo órgano afectado en frecuencia, las manifestaciones clínicas son variables y dependen del perfil del paciente [1].

\section{CASO CLINICO}

Mujer de 23 años de edad, veterinaria, procedente de medio rural de Paraguay que consulta por manchas rojas en piernas y brazos.

\section{Antecedentes Patológicos Personales}

Insuficiencia renal crónica por glomeruloesclerosis difusa de etiología no filiada desde hace 2 años, HTA y anemia de las enfermedades crónicas en tratamiento con prednisona $15 \mathrm{mg} / \mathrm{d}$, bolos mensuales de ciclofosfamida,

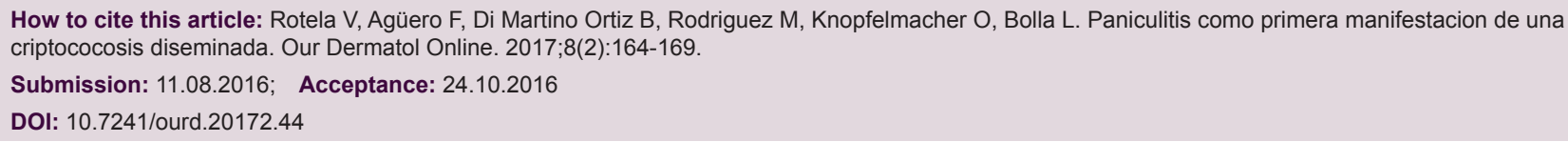


hemodiálisis trisemanal, alfametildopa, furosemida, y eritropoyetina.

\section{Antecedentes De La Enfermedad Actual}

Tres semanas de evolución de manchas rojas en piernas y brazos, que aumentan de tamaño, se endurecen y oscurecen, y manchas oscuras en ambos tobillos. Presenta fiebre de $39^{\circ} \mathrm{C}$ y deposiciones diarreicas. Consulta con facultativo y recibe tratamiento con ciprofloxacina con lo que mejora el cuadro gastrointestinal, pero ante la persistencia del cuadro cutáneo es hospitalizada en Servicio de Medicina Interna de nuestro hospital, desde donde se solicita la evaluación por Dermatología. Niega traumatismos, relación con fármacos, y otras lesiones en piel previamente.

\section{Al Examen Físico}

Nódulos redondeados eritemato-violáceos, de 1 a $3 \mathrm{~cm}$, bordes regulares, limites netos, en cara interna de tercio inferior de piernas y cara interna de brazo derecho. Placa hipercrómica ovalada infiltrada, indurada al tacto, de $3 \times 8 \mathrm{~cm}$, bordes regulares, límites difusos, en cara externa de muslo izquierdo. Múltiples máculas hipercrómicas de entre $1-3 \mathrm{~cm}$ de diámetro, redondeadas, bordes regulares, límites netos, de aspecto residual, localizadas en tobillos (Figs. 1 y 2).

\section{Auxiliares Del Diagnóstico}

\begin{tabular}{lll}
\hline Hb: $7.48 \mathrm{mg} / \mathrm{dl}$ & Hto: $22.5 \mathrm{~g} \%$ & Plaquetas: $160.000 / \mathrm{mm}^{3}$ \\
GB: $12.800 / \mathrm{mm}^{3}$ & N: $86 \%, \mathrm{~L}: 9 \%, \mathrm{M}: 5 \%$ & \\
Glucosa: $104 \mathrm{mg} / \mathrm{dl}$ & Urea: $75 \mathrm{mg} / \mathrm{dl}$ & Cr: $4.46 \mathrm{mg} / \mathrm{dl}$ \\
Factor Reumatoide: Neg & ANA: Negativo & Anti DNA: Negativo \\
Anca-P: Negativo & Anca-C: Negativo & Anti-CCP: Negativo \\
HIV, VDRL: No R & Hepatitis B y C: No R & Citomegalovirus IgG: Neg \\
Toxoplasma Ig G: & Toxoplasma IgM: & Hemocultivos: \\
Positivo & Negativo & Negativos \\
\hline
\end{tabular}

Se procede a toma de muestra para Histopatología y Cultivos de Piel.

En espera de los resultados, la paciente desarrolla alteraciones de conducta, por lo que se realizan estudios imagenológicos, nuevos hemocultivos, y punción lumbar.

\begin{tabular}{lll}
\hline LCR: Límpido & Tinta China LCR: Negativo & RMN de Cráneo: Normal \\
Pandy Negativo & Cultivo de LCR: Negativo & Rx de Torax: Normal \\
Glc: $42 \mathrm{mg} / \mathrm{dl}$ & Hemocultivos: Negativos & \\
Prot: $30 \mathrm{mg} / \mathrm{dl}$ & & \\
Leuc: $75 / \mathrm{mm}^{3}$ & & \\
$62 \%$ PMN; $38 \% \mathrm{MN}$ & \\
\hline
\end{tabular}

\section{Histopatología}

Epidermis y dermis superficial sin alteraciones. Dermis media con escaso infiltrado celular perivascular inespecífico. Dermis profunda e hipodermis con denso infiltrado celular linfo macrofágico. Necrosis licuefactiva tisular con abundantes células inflamatorias entre ellas linfocitos, macrófagos y células gigantes. Estructuras redondeadas birrefringentes, traslúcidas. PAS positivas, levaduriformes, encapsuladas, con halo claro alrededor de núcleo redondeado (Figs. 3 y 4 ).

\section{Diagnóstico Histopatológico}

Criptococosis cutánea.

\section{Cultivo De Piel}

Positivo para Cryptococcus neoformans.

\section{Tratamiento y Evolución}

Fluconazol $400 \mathrm{mg} /$ día IV por dos semanas con mejoría de lesiones cutáneas, luego Fluconazol 200 mg/día

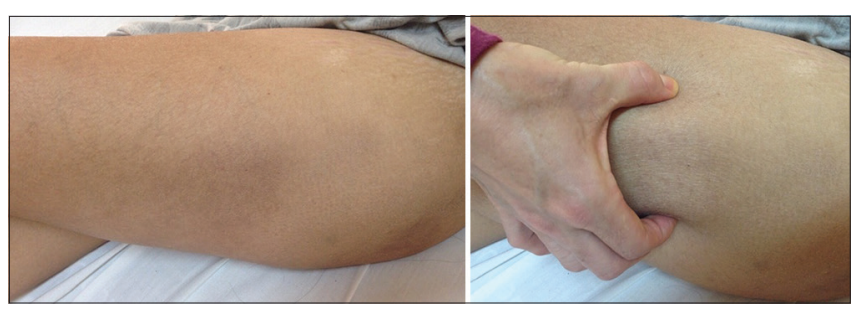

Figure 1: (Clínica) Placa hipercrómica ovalada infiltrada, indurada al tacto, de $3 \times 8 \mathrm{~cm}$, bordes regulares, límites difusos, en cara externa de muslo izquierdo.

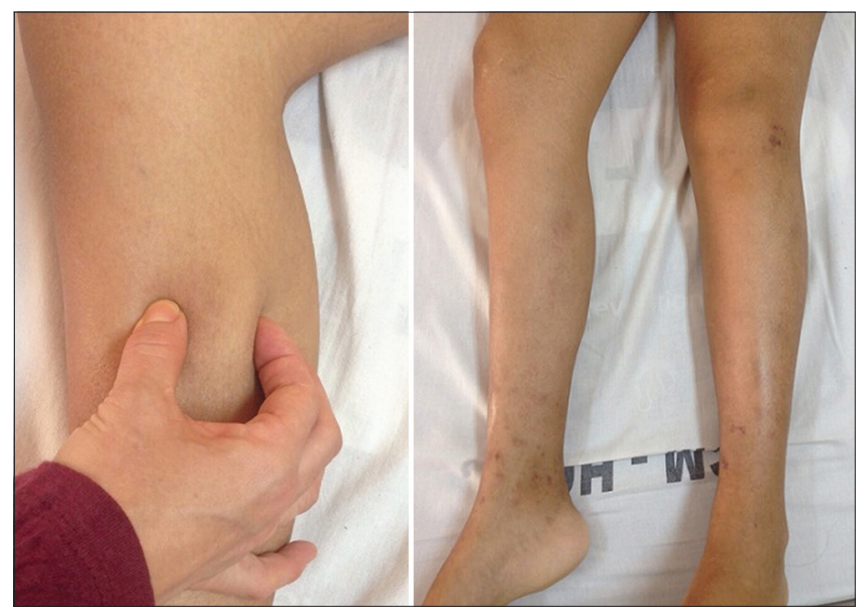

Figure 2: (Clínica) Múltiples máculas hipercrómicas de 1-3 cm de diámetro, redondeadas, bordes regulares, límites netos, de aspecto residual, localizadas en tobillos. Nódulos redondeados eritematovioláceos, de $1 \mathrm{a} 3 \mathrm{~cm}$, en cara interna de tercio inferior de piernas y cara interna de brazo derecho. 
VO, según nuevo cultivo de piel. En caso de presentar empeoramiento o inestabilidad hemodinámica iniciar Anfotericina B.

La paciente fue dada de alta con mejoría de las lesiones en piel, lúcida, con el plan de continuar Fluconazol por un mes y posteriormente realizar nuevo cultivo de piel para valorar continuación del tratamiento (Fig. 5).

Segundo cultivo de piel (al mes): No se observan microorganismos.

Al tercer mes presentó disminución brusca de la agudeza visual y se confirmó endoftalmitis izquierda por Aspergillus sp, por lo que recibió Anfotericina B, y se realizó enucleación del ojo afecto. La paciente fue sometida a transplante renal de donante vivo, con óptima evolución a los 4 meses de seguimiento. Luego de 12 meses de seguimiento no se detectaron otros órganos con invasión por Cryptococcus neoformans.

\section{COMENTARIOS}

La criptococosis es una infección oportunista causada por Cryptococcus neoformans, un hongo encapsulado, que se encuentra en excrementos de aves y murciélagos, frutas, vegetales y en la tierra. La principal puerta de entrada del agente infeccioso es la vía respiratoria. En los pacientes inmunocompetentes, habitualmente la infección se limita a esta localización, desarrollando, en algún caso, una sintomatología y una traducción radiológica escasa con resolución espontánea [1-3].

En los pacientes con inmunosupresión celular, VIH, leucemia o linfoma, trasplantados y pacientes tratados durante un largo periodo de tiempo con esteroides y/o inmunosupresores) la infección puede diseminarse por vía hematógena y afectar a otros órganos, principalmente el sistema nervioso central (SNC) y la piel [2].

Las lesiones cutáneas secundarias aparecen entre el 10 y el $20 \%$ de los casos, entidad conocida como criptococosis cutánea secundaria (CCS).

La criptococosis cutánea primaria (CCP) suele afectar a pacientes inmunodeprimidos, tras inoculación directa. Su diagnóstico se basa en la identificación de C. neoformans en una biopsia o cultivo de la lesión cutánea, asociada a criterios clínicos (la existencia de un síndrome chancriforme) o histológicos (lesiones confinadas a la

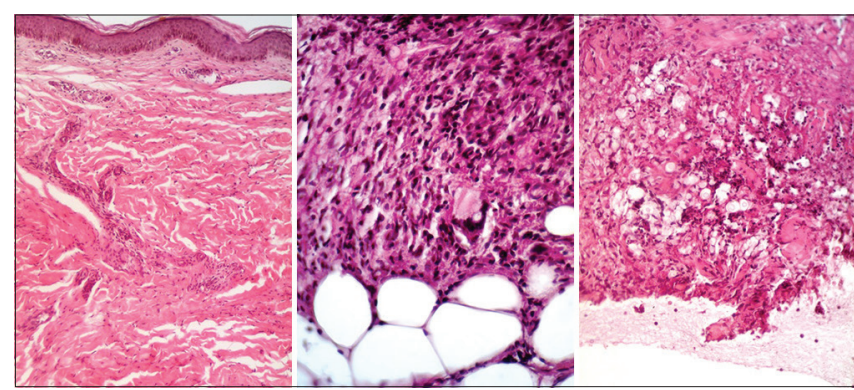

Figure 3: (Histopatología Izq) Epidermis y dermis superficial sin alteraciones. (Centro) Dermis profunda e hipodermis con denso infiltrado celular linfo-macrofágico. Necrosis licuefactiva tisular con abundantes células inflamatorias. (Der) Infiltrado denso a mayor aumento con linfocitos, macrófagos y células gigantes. Estructuras redondeadas birrefringentes, traslúcidas.

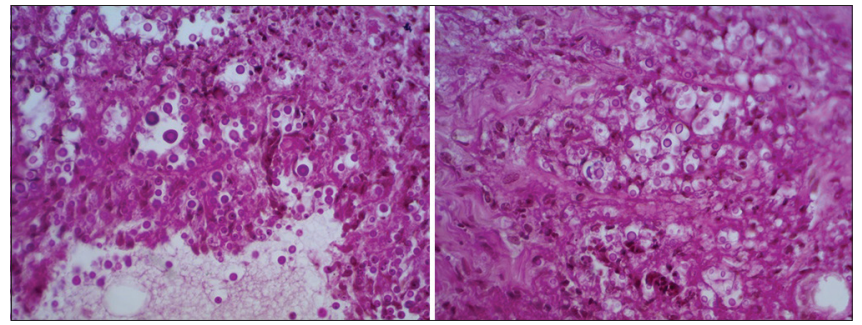

Figure 4: (Histopatología) (Coloración especial de PAS) Dermis profunda e hipodermis con necrosis tisular y denso infiltrado celular linfo-macrofágico, células gigantes, estructuras birrefringentes ovaladas, intracitoplasmáticas, PAS positivas, levaduriformes, encapsuladas, con halo claro alrededor de núcleo redondeado.

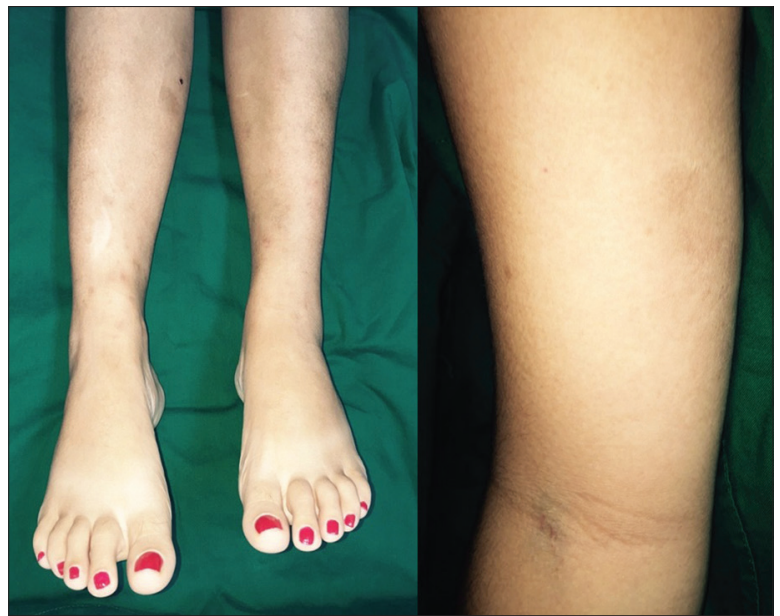

Figure 5: (Evolución) Resolución de lesiones cutáneas al mes de control. Máculas hipercrómicas residuales.

piel y el tejido celular subcutáneo), ambos con ausencia de diseminación, considerando que la linfadenopatía regional no se considera criterio de diseminación [2].

La piel puede afectarse en la Criptococosis diseminada, o en la forma cutánea primaria y es el tercer órgano en frecuencia. En la Criptococosis diseminada se afecta en el 10-20\% de los pacientes, y pueden aparecer 
lesiones en cualquier parte del cuerpo, sobre todo cabeza y cuello [4]. Las manifestaciones cutáneas pueden aparecer meses antes de la afectación de otros órganos, éstas lesiones no son específicas, y pueden ser manifestación de una diseminación generalizada; pápulas, vesículas, pústulas, nódulos subcutáneos que pueden llegar a ulcerarse, úlceras, celulitis, simulando molluscum contagiosum (siendo la forma predominante en pacientes afectos de sida). Muy raramente se han descrito casos de vasculitis necrotizante, casos similares a herpes, pioderma gangrenoso y carcinoma basocelular, nódulos en las extremidades, en el tronco o incluso en el ala nasal, pero no hemos encontrado en la literatura manifestaciones cutáneas que simulen un eritema nodoso, tal y como se ve en nuestro caso $[4,5]$.

El diagnóstico clínico de ambas formas de criptococosis es difícil debido a la inespecificidad de su presentación, por lo que el estudio histopatológico de la piel es importante, ya que tanto el examen convencional con hematoxilina y eosina, así como las tinciones especiales de PAS, plata metenamina y mucicarmín permiten visualizar las esporas hacen sospechar la infección fúngica por cryptococo, sin embargo, el diagnóstico definitivo será microbiológico mediante el cultivo de la muestra seleccionada [4].

El pronóstico es pobre, por su estrecha asociación con la inmunosupresión, y está influenciado por la realización de un diagnóstico precoz y por la instauración rápida de un tratamiento eficaz.
Debe recordarse que los corticoides, fármacos de uso muy habitual en la práctica diaria, son potentes inmunosupresores del sistema inmune humoral y celular, y provocan una mayor predisposición a padecer infecciones por microorganismos oportunistas, circunstancia que obliga a estar atentos ante cualquier síntoma y/o signo, por banal que parezca en los pacientes que los reciben.

El tratamiento de la Criptococosis se resume en la Tabla 1.

Actualmente se cuenta con limitada información sobre la utilidad de los nuevos triazoles, voriconazol y posaconazol para el tratamiento de la criptococosis [6].

\section{CONCLUSION}

Ante la aparición de lesiones en piel en un paciente inmunodeprimido debe plantearse la posibilidad de una infección micótica, aunque las manifestaciones cutáneas sean inespecíficas. El estudio histopatológico de las lesiones cutáneas puede permitir el diagnóstico precoz de algunas micosis profundas, en particular en pacientes de riesgo, mientras se esperan los resultados del cultivo. La celulitis, como la primera manifestación clínica de la infección criptococócica, tal y como ocurrió en nuestra paciente, es inhabitual, generalmente se limita a pacientes inmunodeprimidos y al igual que otras infecciones necrotizantes cursan con alta mortalidad (80\%).

Tabla 1: Tratamiento de la Criptococosis. Modificado de Tello M, Gutiérrez E, y col

\begin{tabular}{|c|c|c|c|}
\hline Criptococosis & Fármaco & Dosis & Tiempo \\
\hline \multirow{2}{*}{$\begin{array}{l}\text { Formas Leves } \\
\text { Sin Compromiso }\end{array}$} & Fluconazol & $400 \mathrm{mg} / \mathrm{d} \mathrm{VO}$ & $6-12$ semanas \\
\hline & Itraconazol* & $400 \mathrm{mg} / \mathrm{d} \mathrm{VO}$ & 6-12 semanas \\
\hline \multicolumn{4}{|l|}{ Formas severas } \\
\hline \multicolumn{4}{|c|}{ Compromiso del SNC } \\
\hline \multicolumn{4}{|c|}{$\mathrm{VIH}+$} \\
\hline \multirow[t]{2}{*}{ Inducción } & Anfotericina B+5-Fluocitosina" & $0,7 \mathrm{mg} / \mathrm{kg} / \mathrm{d}$ EV & 2 semanas \\
\hline & & 100 mg/kg EV & \\
\hline \multirow[t]{2}{*}{ Mantenimiento } & Fluconazol & $400-800 \mathrm{mg} / \mathrm{d}$ VO & 8 semanas \\
\hline & Fluconazol & $200 \mathrm{mg} / \mathrm{d}$ VO & Permanentemente \\
\hline \multicolumn{4}{|l|}{ Formas severas } \\
\hline \multicolumn{4}{|c|}{ Compromiso del SNC } \\
\hline \multicolumn{4}{|c|}{$\mathrm{VIH}-$} \\
\hline Alternativas $^{\circ}$ & Anfotericina B+5-fluocitosina" & & $4-6$ semanas \\
\hline Inducción & Anfotericina B+Fluconazol & $\begin{array}{l}0,7 \mathrm{mg} / \mathrm{kg} / \mathrm{d} \text { EV } \\
800 \mathrm{mg} / \mathrm{día} \text { VO }\end{array}$ & 2 semanas \\
\hline Mantenimiento & Fluconazol & $\begin{array}{l}400-800 \text { mg/día } \\
\text { VO }\end{array}$ & $8-10$ semanas \\
\hline
\end{tabular}

*Menos efectivo, "La combinación ayuda a más rápida negativización del LCR, ํㅡ caso de no contar con 5-Fluocitosina, `Hasta la negativización del LCR 
La importancia de conocer las manifestaciones cutáneas de esta infección se basa en que dichas lesiones pueden preceder incluso hasta dos años la afectación por Cryptococus neoformas de otros órganos o sistemas, de curso insidioso y oligosintomático, como la afectación pulmonar o neurológica, éstas manifestaciones pueden preceder.

La fácil accesibilidad para realizar una biopsia cutánea, mínimamente invasiva y con gran rentabilidad diagnóstica, permite obtener muestras para el cultivo microbiológico y para el estudio histopatológico, en muchas ocasiones imprescindibles debido a la inespecificidad y al polimorfismo clínico.

Esta infección debe considerarse en el diagnóstico diferencial de la celulitis/hipodermitis necrotizante en pacientes inmunodeprimidos, la afectación de la piel como la primera manifestación clínica de la infección criptococócica diseminada es rara, siendo de mal pronóstico por lo que debe realizarse un precoz diagnóstico y tratamiento.

\section{BIBLIOGRAFIA}

1. Bordel Gómez M. Celulitis necrotizante como primera manifestación de una criptococosis diseminada. Actas Dermosifiliográf. 2011;102:297-307.

2. Ballestero M, García I, Daudén E, Sánchez-Pérez J, Iscar T, Fraga J, et al. Criptococosis cutánea primaria asociada a oncotaxia en un paciente inmunodeprimido. Actas Dermosifiliográf. 2004;95:574-7.

3. Ikeda T, Kaminaka C, Yamamoto Y, Furukawa F. Disseminated Cryptococcosis-Induced Skin Ulcers in a Patient with Autoimmune Hepatitis. Case Rep Dermatol. 2014;6:98-102.

4. Ruíz V. Nódulos cutáneos similares a Eritema nodoso como manifestación de criptococosis cutánea diseminada. Actas Dermosifiliográf. 2011;102:221-37.

5. Haight D, Lowella E, Greene J, Sandin R, DeGregorio R, Spiers A. Case report: Cutaneous manifestations of Crytococcosis. Am J Med Sci. 1994;308:192-5.

6. Tello M, Gutiérrez E, Béjar V, Carlos Galarza C, Ramos W, OrtegaLoayza A. Criptococosis. Rev Méd Risaralda. 2013;19:147-53.

Copyright by Verónica Rotela, et al. This is an open-access article distributed under the terms of the Creative Commons Attribution License, which permits unrestricted use, distribution, and reproduction in any medium, provided the original author and source are credited.

Source of Support: Nil, Conflict of Interest: None declared. 